\title{
Maus tratos infantis: avaliação do currículo dos cursos em odontologia
}

Stephanye Pinto Biss"; João Gilberto Duda*; Paulo H. Tomazinhoo**; Eduardo Pizzatto ${ }^{* * * *}$; Estela Maris Losso ${ }^{* * * * *}$

* Aluna do curso de graduação em Odontologia, Universidade Positivo, Curitiba, PR. Bolsista do Programa de Iniciação Científica (PIBIC) do CNPq

** Especialista em Odontopediatria. Aluno do Curso de Mestrado Profissional em Odontologia Clínica da Universidade Positivo, Curitiba, PR

*** Mestre em Microbiologia. Professor do Curso de Odontologia da Universidade Positivo, Curitiba, PR

**** Doutor em Saúde Coletiva, Professor do Curso de Odontologia e do Curso de Mestrado Profissional em Odontologia da Universidade Positivo, Curitiba, PR

***** Doutora em Odontopediatria, Professora do Curso de Odontologia e do Curso de Mestrado Profissional em Odontologia da Universidade Positivo, Curitiba, PR

\section{RESUMO}

O objetivo desta pesquisa foi avaliar se o tema maus tratos infantis é abordado nos cursos de graduação em Odontologia no Brasil. Trata-se de um estudo exploratório transversal descritivo, desenvolvido com todos os cursos de Odontologia do país. Foram enviados questionários aos 201 coordenadores e 174 foram recebidos, porém somente 28 retornaram respondidos (taxa de resposta de 16,09\%). Destes, 19 (67,8\%) correspondem a instituições privadas, 06 (21,4\%) públicas e 03 (10,7\%) de gestão compartilhada. A temática é abordada em 21 cursos (75\%), principalmente nas disciplinas de odontopediatria, odontologia legal e saúde coletiva. Quanto à carga horária destinada a este tema, 16 cursos $(21,19 \%)$ afirmaram ser igual ou menor que 08 horas e 14 coordenadores $(66,66 \%)$ declararam ser obrigatória a abordagem de tal tema dentro do conteúdo programático do curso. Devido à impor-

tância do tema maus tratos infantis e frente aos resultados deste estudo, é pertinente concluir que este assunto tem sido trabalhado de forma incipiente nos cursos de odontologia no Brasil.

Descritores: Violência. Criança. Educação em Odontologia.

\section{INTRODUÇÃO}

As Diretrizes Curriculares Nacionais do Curso de Graduação em Odontologia estabelecem que os cursos de graduação em odontologia no Brasil devem formar profissionais generalistas, com formação abrangente e humanista, tendo uma conduta mais reflexiva e crítica, fugindo do padrão tecnicista até então difundido, para que ele esteja apto a desenvolver um papel transformador na sociedade. Sendo assim, cria-se a necessidade de repensar o projeto pedagógico dos cursos e investir em sua adequação às necessidades atuais ${ }^{1}$. Neste contexto surge a necessidade de capacitar o cirurgião dentista quanto ao reconhecimento e conduta frete a ocorrência de maus tratos infantis.

De acordo com o Ministério da Saú$\mathrm{de}^{2}$ abuso e negligência infantil é toda e qualquer ação ou omissão que prejudique o bem-estar físico e psicológico, da liberdade e 
do direito ao desenvolvimento integral das crianças. Este abuso pode ser físico, sexual, psicológico ou mesmo negligência. A falta de condições básicas para ao desenvolvimento da criança é considerada negligência, sendo que a desatenção quanto à saúde bucal da criança também pode ser caraterizada como negligência ${ }^{3,4}$.

Como profissionais de saúde, os cirurgiões-dentistas exercem papel fundamental no reconhecimento e na denúncia dos maus tratos, posto que de 50 a $65 \%$ das lesões ocorrem na região de cabeça e pescoço, sendo muitas vezes lesões orofaciais ${ }^{5,6}$.

No Brasil, o Estatuto da Criança e do Adolescente estabelece que todos os cidadãos devem notificar os casos suspeitos de maus tratos ao juizado de menores, a fim de proteger os direitos destes indivíduos. $\mathrm{O}$ artigo 245 diz que os médicos, professores e responsáveis por estabelecimentos de atenção à saúde, creches, pré-escola e escola podem ser punidos por não comunicarem a suspeita de violência à autoridade competente ${ }^{7}$. Assim sendo, o papel do profissional de saúde assume elevada importância.

Estudos realizados no município de Curitiba revelaram que os odontopediatras e os profissionais da Rede Pública têm dificuldade no diagnóstico e conduta dos casos suspeitos e que houve subnotificação dos casos mesmo sabendo onde notificar ${ }^{8,9}$.

Tanto na literatura brasileira como na internacional, os vários estudos mostram que há necessidade de mais orientação aos cirurgiões dentistas para o diagnóstico e conduta de maus tratos infantis e também para a notificação dos mesmos ${ }^{8-15}$. Quando verificado o interesse em explorar mais o assunto de maus tratos infantis, os profissionais de saúde se mostraram interessados e os odontopediatras afirmaram que gostariam de receber mais informações sobre maus tratos, as quais foram praticamente ausentes na graduação, $\mathrm{e}$ que quando presentes eram insuficientes ${ }^{8,9}$.

Estudo realizado nos Estados Unidos abordou a importância da educação dos alunos de odontologia com o objetivo de estimular a sua capacidade de percepção de abuso em crianças e adolescentes, trabalhando com um protocolo apropriado para melhor conduta dos estudantes ${ }^{16}$.

Não foram encontradas pesquisas realizadas no Brasil verificando se o tema maus tratos infantis faz parte do currículo de formação do cirurgião dentista. Diante da importância do conhecimento deste assunto por parte do cirurgião dentista, o presente estudo tem por objetivo verificar se o tema maus tratos na infância e adolescência é abordado nos cursos de graduação em odontologia do Brasil.

\section{MATERIAIS E MÉTODOS}

Esta pesquisa foi aprovada pelo Comitê de Ética e Pesquisa da Universidade Positivo, parecer 381622.

Realizou-se um estudo observacional, transversal e quantitativo, onde foram enviados questionários autoaplicáveis contendo questões estruturadas e semiestruturadas aos 201 coordenadores dos cursos de odontologia do Brasil. Para obtenção das informações das instituições de ensino superior do Brasil que oferecem o curso de Odontologia, foi utilizada a lista de cursos disponibilizados pelo Conselho Federal de Odontologia (CFO) em seu endereço eletrônico (http://cfo.org.br/servicos-e-consultas/faculda des/).

O questionário (Quadro 1) foi enviado utilizando a ferramenta Google Drive, juntamente com o questionário, foi enviado o termo de consentimento livre e esclarecido. O teste piloto foi realizado com dez professores do Curso de Odontologia da própria Universidade Positivo. Em seguida, foram enviados os questionários aos coordenadores dos Cur-15(1): 55-62, 2015. 
sos de Odontologia do Brasil.

Quadro 1 - Questionário utilizado na pesquisa

1. Você concorda em participar desse estudo? ( ) Sim ( ) Não

2. Nome da Instituição.

3. Em que região do Brasil se localiza a Instituição?

4. A Instituição é: ( ) Pública ( ) Particular ( ) Gestão compartilhada.

5. O assunto maus tratos contra crianças e adolescentes é abordado na graduação? ( )Sim ( ) Não

6. Se sim, em quais disciplinas?

7. Em que período este assunto é trabalhado?

8. Quantas horas são trabalhadas esse assunto?

9. Como o tema é trabalhado? ( ) Aula teórica ( ) Discussão de casos ( ) Trabalhos ( ) Eventos ( ) Palestras, seminários, workshops ( ) Outro

10. Qual o tipo de vinculação do conteúdo no currículo? ( ) Obrigatória ( ) Optativa

11. Em sua opinião, este assunto deve ser obrigatório no Curso de Odontologia? Por quê?

\section{RESULTADOS}

Os questionários foram enviados em três momentos, sendo o primeiro no mês de novembro de 2013. Dos 201 questionários enviados aos coordenadores, 11 responderam. Em janeiro de 2014, realizou-se o segundo envio, obtendo-se mais 9 questionários respondidos. Já em fevereiro de 2014, o retorno foi de 7 questionários, totalizando 28 questionários respondidos, com uma taxa de resposta de 16,09\%. Destas 21,4\% ( $\mathrm{n}=6)$ eram instituições públicas $67,8 \%(n=19)$ privadas e $10,7 \%(n=3)$ de gestão compartilhada, sendo que $75 \%$ delas relataram abordar o assunto ao longo da graduação.

O Gráfico 1 representa a contribuição de cada região nas respostas. As que tiveram maiores taxas de respostas foram a região Sul (32,14\%) e Sudeste (35,71\%). 
Gráfico 1 - Respondendes por região (n=28)



O Gráfico 2 representa as disciplinas odontopediatria (47\%), odontologia legal que abordagem o tema maus tratos na gradu- $\quad$ (20,5\%) e saúde coletiva (17,64\%). ação. As disciplinas mais citadas foram a de



O Gráfico 3 representa o período em citado, com 26\% das respostas, seguido pelo que o tema maus tratos é ministrado. O sétimo período com 17,39\%. oitavo período da graduação é o mais 
Gráfico 3 - Período nos quais o tema maus tratos é ministrado (n=46). Mais de uma resposta era possível

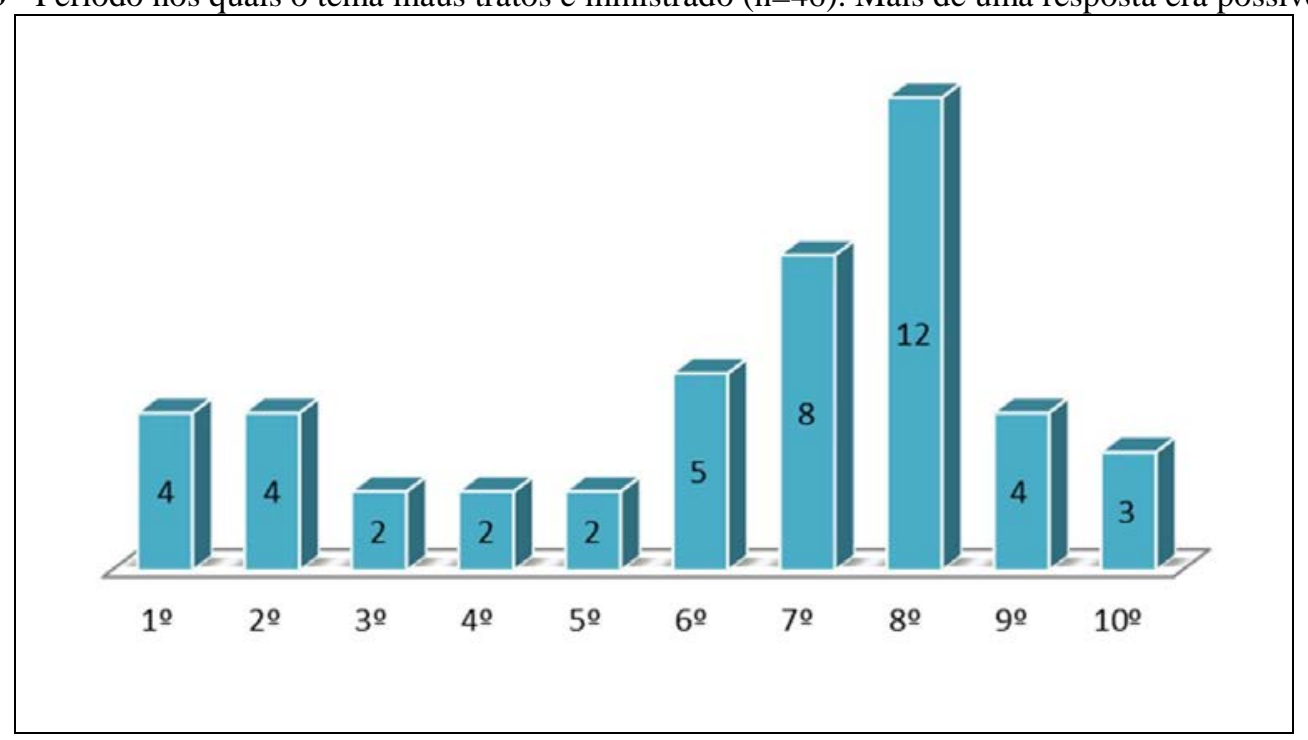

Mais de $46 \%$ dos respondentes afirmaram que a disciplina maus tratos deveria ser obrigatória na graduação. O Gráfico 4 representa os motivos dos quais o tema maus tratos deveria ser obrigatório na graduação. Os mais citados foram a falta de conhecimento, com $46,8 \%$ e necessidade de educação, com $19 \%$.

Gráfico 4 - Distribuição das respostas em relação à obrigatoriedade do tema maus tratos (n=47). Mais de uma resposta era possível

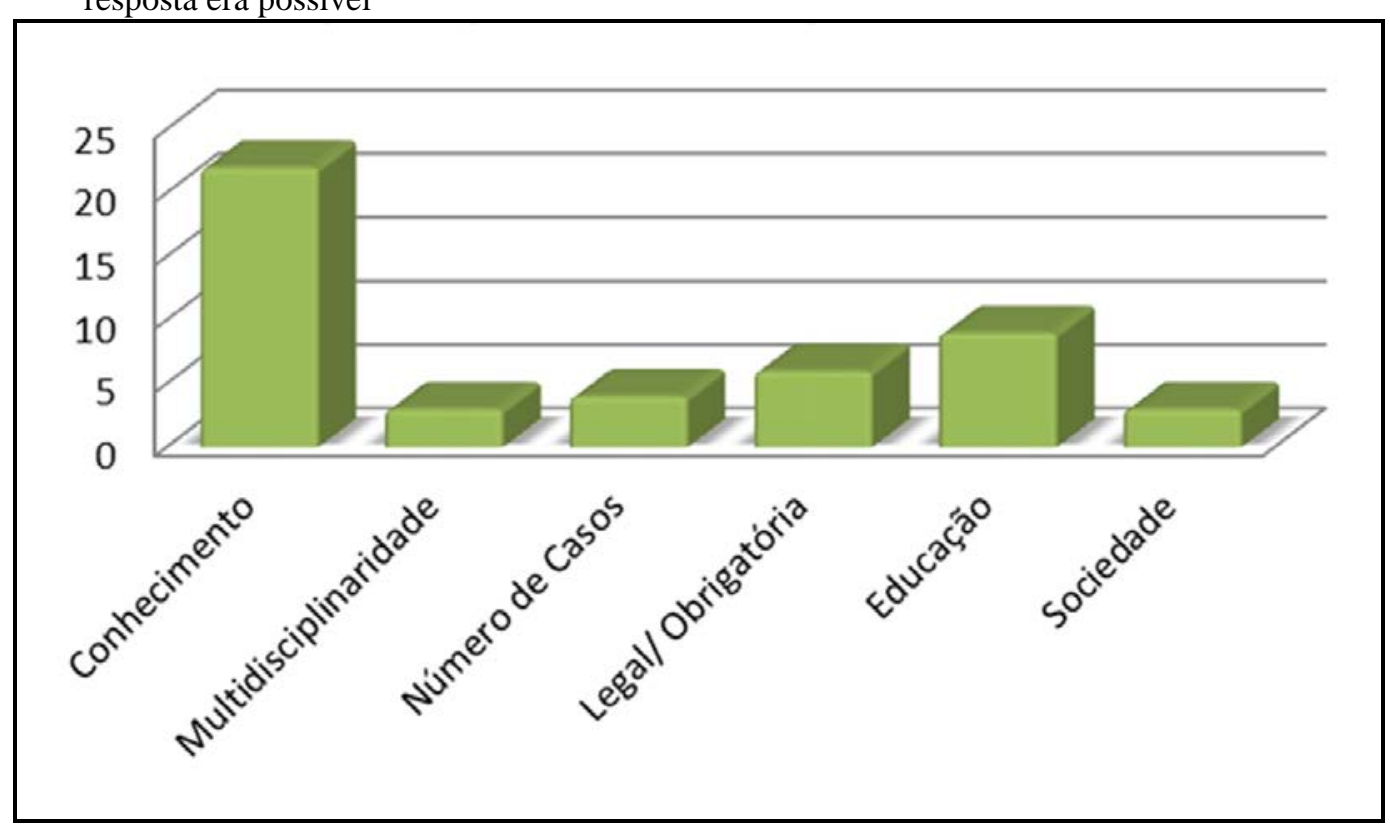

A carga horária destinada à discussão do tema maus tratos foi, em $24 \%(n=5)$ das instituições respondentes, mais de 8 horas e, em $76 \%(n=16)$, menos de 8 horas durante todo o curso de odontologia. As aulas teóricas foram o modo didático mais usado com
46,15\% ( $n=18)$, seguido por discussão de casos 30,7\% $(n=12)$, trabalhos em grupos $12,8 \%(n=5)$ e eventos $10,2 \%(n=4)$. Mais de uma resposta era possível com um total de 39 respostas. 


\section{DISCUSSÃO}

No período da pesquisa estavam ativos 201 cursos de graduação em odontologia no Brasil, com maior concentração nas Regiões Sudeste e Sul. As regiões que obtiveram a maior taxa de resposta foram respectivamente Sudeste e Sul, com 35,71\% e 32,14\%, respectivamente. A taxa de resposta dos questionários foi de 16,09\%, sinalizando a falta de interesse dos gestores dos cursos de graduação sobre pesquisas científicas ou mesmo pelo assunto.

Mais de 50\% dos participantes acreditam que o assunto deve ser optativo, porém estudos mostram a necessidade de mais conhecimento dos cirurgiões dentistas devido à baixa notificação de casos suspeitos de maus tratos $^{8-10,13-15}$. El Sarraf et al. ${ }^{8}$ verificaram que 88\% dos profissionais pesquisados tiveram pouca informação sobre o assunto na graduação e os resultados mostraram que houve subnotificação dos casos suspeitos de maus tratos infantis em pacientes por eles atendidos. Esses dados demonstram a importância da inclusão do tema na grade curricular dos cursos de graduação em odontologia.

O presente estudo mostrou que 0 assunto é abordado principalmente no final do curso, nas disciplinas de odontopediatria, provavelmente por ser o momento de maior atendimento a crianças e adolescentes. Devese pensar na possibilidade da abordagem do tema durante todo o curso da graduação. Ivanoff $^{16}$ fez uma proposta de trabalhar o tema durante o curso de odontologia e para isto a dividiu em fases. A primeira fase (primeiro e segundo anos) implica no ensino dos indicadores físicos e comportamentais. Fazer a distinção entre abuso físico e sexual e entre abuso infantil e negligência. Na segunda fase há uma combinação de palestras e casos antes de os alunos entrarem na clínica no terceiro ano. Já na fase três, é aonde ocorre a simulação de relato de casos às autoridades, a res- ponsabilidade legal do estudante é dada a prova. Na fase quatro, o curso proporciona ao aluno prática real de ter um contato com os pais e com a criança ${ }^{16}$.

Os resultados mostraram que $76 \%$ dos cursos trabalhavam o tema em menos de oito horas no curso todo. Já Ivanoff ${ }^{16}$ verificou que na graduação não havia mais que três horas oferecidas para este tema, mas a sua proposta seria de, pelo menos, quatorze horas.

Apesar de o cirurgião-dentista ter a obrigação ética e legal de notificar os casos suspeitos de maus tratos infantis, e de estar numa posição propicia para perceber e diagnosticar os casos suspeitos de maus tratos, ainda ocorrem muitos casos de subnotificação neste grupo de profissionais.

Estudo realizado com endodontistas verificou que apenas $12 \%$ dos casos suspeitos foram notificados as autoridades ${ }^{17}$, estes afirmaram também que tiveram pouca ou nenhuma abordagem deste tema na graduação e na pós-graduação. Resposta semelhante foi obtida no trabalho realizado com odontopediatras $^{8}$. Algumas situações favorecem as sub notificações de casos suspeitos de maus tratos contra crianças, como medo de perder pacientes, falta de confiança no serviço de proteção à criança e aos jovens, medo de lidar com os pais, incerteza do diagnóstico e conhecimento de verdadeira responsabilidade em denunciar os casos suspeitos de maus tratos $^{9,13}$.

O cirurgião dentista deve participar na prevenção secundária que é a detecção de crianças em situação de risco ${ }^{18}$, contemplando o é proposto pelas Diretrizes Curriculares Nacionais: "O Curso de Graduação em Odontologia tem como perfil do formando egresso/profissional o Cirurgião Dentista, com formação generalista, humanista, crítica e reflexiva, para atuar em todos os níveis de atenção à saúde, com base no rigor técnico e 15(1): 55-62, 2015. 
científico. Capacitado ao exercício de atividades referentes à saúde bucal da população, pautado em princípios éticos, legais e na compreensão da realidade social, cultural e econômica do seu meio, dirigindo sua atuação para a transformação da realidade em benefício da sociedade"19. Isto demonstra a necessidade de trabalhar o tema na formação do aluno de odontologia, evidenciando o seu papel como futuro profissional de saúde. Além disso, este tema requer a continuidade de atualização após a graduação, em congressos e outros eventos.

\section{CONCLUSÃO}

Devido à importância do tema maus tratos infantis e frente aos resultados deste estudo, é pertinente concluir que o assunto tem sido trabalhado de forma incipiente nos Cursos de Odontologia no Brasil.

\section{REFERÊNCIAS}

1. Musse JO, Boing AF, Martino FS, Silva RHA, Vaccarezza GF, Ramos DLP. O Ensino da bioética nos cursos de graduação em odontologia do estado de São Paulo. Arq Ciênc Saúde 2007 janmar;14(1):13-6

2. Brasil. Ministério da Saúde. Secretaria de Assistência à Saúde.Notificação de maus-tratos contra crianças e adolescentes pelos profissionais de saúde.Um passo a mais na cidadania em saúde / Ministério da Saúde, Secretaria de Assistência à Saúde. - Brasília: Ministério da Saúde, 2002.

3. WHO. Preventing child maltreatment: a guide to taking action and generating evidence /World Health Organization and International Society for Prevention of Child Abuse and Neglect. 2006.p89

4. American Academy of Pediatrics Committee on Child Abuse and Neglect and the American Academy of Pediatric
Dentistry. Guide Orient Dent Aspects Child Abuse Neglect 2010; 33(6):11-2.

5. Valente LA, Dalledone M, Pizzatto E, Zaiter W, de Souza JF, Losso EM Domestic violence against children and adolescents: prevalence of physical injuries in a southern Brazilian metropolis Braz Dent J. 2015 Jan-Feb; 26(1):55-60.

6. Cavalcanti AL. Prevalence and characteristics of injuries to the head and orofacial region in physically abused children and adolescents - a retrospective study in a city of the Northeast of Brazil. Dental Traum. 2010 Apr; 26: 149-53.

7. Brasil. Estatuto da criança e adolescente. Lei $n^{\circ}$. 8.069 de 13 de julho de 1990.

8. El Sarraf MC, Marengo G, Correr GM, Pizzatto E, Losso EM. Physical child abuse: perception, diagnosis, and management by southern Brazilian pediatric dentists. Pediatr Dent. 2012 Jul-Aug; 34(4): 72-6.

9. Paola, APB. Maus-tratos na infância e adolescência: percepção e conduta dos cirurgiões dentistas da Rede Pública Municipal de Curitiba. 2012. Dissertação (Mestrado Profissional em Clínicas Odontológicas) - Universidade Positivo. p.55.

10. Cairns AM, Mok JYQ, Welbury RR. The dental practitioner and child protection in Scotland. Brit Dent. J 2005 Oct;199(8):517-20.

11. Thomas JE, Straffon L, Inglehart MR. Knowledge and professional experiences concerning child abuse: an analysis of provider and student responses. Pediatr Dent. 2006 Sep-Oct; 28(5):438-44.

12. Manea S, Favero GA, Stellini E, Romoli L, Mazzucato M, Facchin P. Dentists` perceptions, attitudes, knowledge, and experience about child abuse and neglect in Northeast Italy. J Clin Pediatr Dent 2007 Fall;32(1):19-25 
13. Granville-Gracia AF, Silva MJF, Menezes VA. Maus-tratos a criança e adolescentes: Um estudo em São Bento do Uma, PE, Brasil. Pesq Bras Odontoped Clin Integr 2008 8(3);301-7.

14. Uldum B, Christensen HN, Welbury R, Poulsen S. Danish dentistsand dental hygienists knowledge of and experience with suspicion of child abuse or neglect. Int J Paediatr Dent 2010 Sep: 20:361-5.

15. Azevedo MS, Goettems ML, Brito A, Possebon AP, Domingues J, Demarco FF, Torriani DD. Child maltreatment: a survey of dentists in southern Brazil. Braz Oral Res 2012 Jan-Feb; 26(1):5-11.

16. Ivanoff CS, Hottel TL. Comprehensive training in suspected child abuse and neglect for dental students: a hybrid curriculum. J Dent Educ. 2013 Jun;77(6):695705.

17. Losso EM, Marengo G, El Sarraf MCF, Baratto-Filho F. Child abuse: perception and management of brazilian endodontists.. RSBO. Revista Sul-Brasileira de Odontologia, 2012 jan-mar: 9 (1): 62-6.

18. Martins CBG, Jorge MHPM. Maustratos infantis: um resgate da história e das políticas de proteção. Acta Paul Enferm. 2010 23(3): 423-8.

19. Brasil. Conselho Nacional de Educação. 2002. Disponível em http://portal.mec.gov.br/cne/arquivos/pdf /CES032002.pdf. Acesso em 06 de agosto de 2014.

\section{AGRADECIMENTOS}

Ao CNPQ pela concessão da bolsa PI-
The aim of this study was to evaluate whether the theme child abuse is addressed in the undergraduate dentistry in Brazil. This is a cross-sectional exploratory study, in which all universities in the country with the course of dentistry were questioned. Questionnaires were forwarded to all 201 coordinators of education of dentistry in Brazil, which consist in all existing courses at the time of the research. 174 questionnaires were received by the coordinators; however, only 28 were answered (response rate of $16.09 \%$ ). Of these, 19 (67.8\%) were private institutions, 06 (21.4\%) public and 03 (10.7\%) were joint management. The theme is approached by 21 institutions (75\%), mainly in the disciplines of Dentistry, Forensic Dentistry and Public Health. Regarding the amount of hours designated for this theme, 16 institutions (21.19\%) mentioned to reserve 08 hours or less during the course, on the other hand, 14 institutions (66.66\%) stated that they were required to approach this issue within the course. Due to the importance of the theme child abuse and compared to the results of this study, it seems reasonable to conclude that this matter has been worked very incipient in Dental Courses in Brazil.

Descriptors: Violence. Child. Education. Dental.

Correspondência para:

Prof $^{a}$ Dr $^{a}$ Estela Maris Losso

e-mail: lossoem@gmail.com

Rua Prof. Pedro Viriato Parigot de Souza, 5300 - Campo Comprido 81280-330 -

Curitiba/PR BIC.

\section{ABSTRACT \\ Child abuse: curriculum evaluation of dental courses in Brazil}

